CAUSATION

\title{
Review: previous caesarean delivery or abortion increases the risk of placenta praevia
}

\author{
Ananth CV,Smulian JC, Vintzileos AM. The association of placenta previa with history of cesarean delivery and abortion: a \\ metaanalysis. Am J Obstet Gynecol 1997;177:1071-8.
}

\section{Question}

Does a history of caesarean deliveries, spontaneous abortions, or induced abortions increase the risk of placenta praevia?

\section{Data sources}

Studies were identified by searching Medline databases using the keywords placenta praevia, placental disorders, antepartum haemorrhage, antepartum bleeding, caesarean delivery, caesarean section, uterine surgery, spontaneous abortion, induced abortion, and elective abortion; and by scanning bibliographies of relevant papers.

\section{Study selection}

Studies were selected if they provided quantitative analysis of placenta praevia and were published in English between 1950 and 1996. Studies on placental abruption and case reports were excluded.

\section{Data extraction}

Data were extracted on the total number of pregnancies; the number of pregnancies complicated by placenta praevia; previous caesarean deliveries, spontaneous abortions, and induced abortions; study design; and the country in which the study was run. Odds ratios (ORs) were calculated using fixed and random effects models with results from random effects models considered to be more reliable due to evidence of heterogeneity among studies.

\section{Main results}

In 36 studies, 13992 of 3700000 women who were pregnant had placenta praevia. The reported incidence was approximately 1 in 200 pregnancies (range $0.28 \%$ to $1.96 \%$ ). 8 cohort and 7 case control studies showed an increased risk of placenta praevia for women who had previous caesarean deliveries (OR 2.6, 95\% CI 2.3 to 3.0). Case control studies showed a larger effect for previous caesarean deliveries (OR 3.8, CI 2.3 to 6.4) than did cohort studies (OR 2.4, CI 2.1 to 2.8), and there was little difference between American studies and other studies. Subgroup analysis of 4 relevant studies showed that the risk of placenta praevia increased with the number of previous caesarean deliveries (OR 4.5, CI 3.6 to 5.5 for women who had 1 previous caesarean delivery; OR 7.4, CI 7.1 to 7.7 for women who had 2; OR 6.5, CI 3.6 to 11.6 for women who had 3; OR 44.9, CI 13.5 to 149.5 for women who had $\geqslant 4$ ). 1 cohort and 4 case control studies showed an increased risk of placenta praevia for women who had $\geqslant 1$ previous spontaneous abortion (OR 1.7, CI 1.0 to 2.9). 5 case control studies and 1 cohort study showed an increased risk of placenta praevia for women who had $\geqslant 1$ previous induced abortion (OR 1.6, CI 1.0 to 2.6). 1 cohort study showed that the risk of placenta praevia increased with the number of previous abortions, either spontaneous or induced (OR 1.6, CI 1.3 to 1.8 for women who had 1 induced abortion; OR 2.3, CI 1.8 to 3.0 for women who had 2; OR 3.7 , CI 2.7 to 5.2 for women who had $\geqslant 3$ ).

\section{Conclusions}

Women who have had a previous caesarean delivery or a spontaneous or induced abortion have a greater risk of developing placenta praevia. The risk increases with the number of previous caesarean deliveries or abortions.

Source of funding: no external funding.

For article reprint: $\operatorname{Dr} C \mathrm{~V}$ Ananth, The Center for Perinatal Health Initiatives, Department of Obstetrics, Gynecology and Reproductive Sciences, University of Medicine and Dentistry of New Jersey, Robert Wood Johnson Medical School, 125 Paterson Street, New Brunswick, NJ 08901-1977, USA.Fax +1 7322356627

\section{Commentary}

Current research on the management of childbirth suggests greater caution in the use of caesarean delivery, and expresses concern over high caesarean delivery rates and reluctance by some clinicians to adopt strategies encouraging vaginal birth after caesarean delivery. ${ }^{1-3}$ Ananth et al provide further evidence for caution when deciding whether to perform primary and repeat caesarean delivery. They present a meta-analysis showing an increased incidence of placenta praevia in pregnancies after caesarean delivery and abortion.

The review suggests that women who have experienced caesarean delivery may be 2-3 times more likely to develop placenta praevia than other women, and that this increased risk is further exacerbated by additional caesarean deliveries. The authors calculate that if caesarean delivery rates could be halved (ie, from $20 \%$ to $10 \%$ ), then $14 \%$ of all placenta praevia cases would not occur. This is important new evidence to support clinical policies which aim to reduce primary and repeat caesarean delivery rates. The authors caution, however, that improvements over time in the detection of placenta praevia via ultrasound may exert an effect on the apparent positive links between rates of caesarean delivery and placenta praevia. They also note that as maternal age and parity have been identified as risk factors for placenta praevia, differences in the number of previous caesarean deliveries could be partly due to age and parity differences.

The study suggests that women who have had either spontaneous or induced abortions have a substantially increased risk of developing placenta praevia in subsequent pregnancies. Inclusion of such information when counselling women before an induced abortion may therefore be justified. Implications exist also for those providing antenatal care. Women with a history of either caesarean delivery or abortion could be considered to be at higher risk for placenta praevia in pregnancy. Further evaluation of the potential costs and benefits of additional screening would be necessary before new clinical policies are recommended.

Results presented in this study may not single handedly influence practice patterns in childbirth management, but when combined with evidence from other studies, the case for decreasing rates of caesarean delivery is further strengthened.

\section{Allison Shorten, RN, CM, MSc Lecturer in Midwifery University of Wollongong New South Wales, Australia}

1 Lomas J, Enkin M. In: Chalmers I, Enkin M, Keirse MJ, editors. Effective care in pregnancy and childbirth. Oxford: Medical Publications, 1989:1182-95.

2 Paul RH, Miller DA. Am J Obstet Gynecol 1995;172:1903-7.

3 Stafford RS. JAMA 1990;263:683-7. 